\title{
A DYNAMIC-BOOSTER AND ATTENDANCE-TRACKING APP FOR INTERACTIVE CLASSROOM TEACHING
}

\author{
Alberto Brunete ${ }^{1}$, Raquel Cedazo ${ }^{2}$ \\ ${ }^{1}$ Centre for Automation and Robotics (CAR UPM-CSIC), Universidad Politécnica de Madrid, \\ C/ Ronda de Valencia, 3, 28012 Madrid (Spain) \\ ${ }^{2}$ High School of Technical Industrial and Design Engineering. Universidad Politecnica de \\ Madrid, C/ Ronda de Valencia, 3, 28012 Madrid (Spain)
}

\begin{abstract}
This paper presents an app whose main aim is to improve the user experience in face-to-face classes, both for lecturers and students. This app allows the lecturer to have more control over the class, registering and visualizing the student attendance and performance, and allows the students to participate more actively during the sessions through live questions.

After installing a QR code on the classroom tables, the student is able to check-in with the App when arriving to class. A map of the class is created automatically for the lecturer with names, photos and the location of each student. The lecturer uses this map (running in a tablet or computer) to know the names of the students, to pose questions, to see their statistics and attendance, and to interact on a more personal level (especially relevant for large classes over 100 students).

The app allows the lecturer to propose questions, tests and exercises that the students respond individually and confidentially through the same app. This allows the lecturer to have feedback in real time of the degree of attention and understanding of the students. Student answers could be used to make rankings, and therefore award prizes according to each student performance (gamification techniques).
\end{abstract}

Keywords: Attendance-tracking, dynamic teaching, interactive classroom

\section{INTRODUCTION}

Throughout the years, smartphones have become one of the most important devices of our daily life. However, regarding education, we are trying to eradicate it from the classrooms because of the tendency to distract students.

But in this project, we have tried to see smartphones not as an enemy, but as a friend, as a tool with increasing potential and from which teaching can also benefit through new innovative methodologies.

Therefore, the motivation of this project is to convert smartphones and tablets in a platform to boost the classes, motivate students to participate without having that fear of making mistakes in public, allow the teacher to have a better control of the class, and be able to keep track of the attendance in the classes automatically.

If we review the literature, there are similar applications and even a comparison of them [1]. Probably Kahoot! [2] is the most frequently used question-and-answer web tool to interact with students and have an immediate feedback of the answers. Today it has more than 30 million users worldwide and is used in both academic and professional fields. Plickers [3] [4] is another of these applications. In this case it is the teacher who has the mobile device, while the students have response cards that the app itself identifies and, from those answers, extracts the results. Quizizz [5] is an app whose operation is similar to Kahoot! but it allows to personalize the questions to create own contests or examinations in a playful way.

This article explains how the developed application works, the technology used and the resources necessary for its implementation. Finally, the conclusions of the work are exposed. 


\section{METHODOLOGY}

In this section the general objectives, the technologies used, a general overview of the system and a description of the functionalities will be explained.

\subsection{Objectives}

The objectives of the applications are the following:

1. Booster classes, by formulating questions, tests, games, etc. in class.

2. Give quick feedback to students on his learning process, since they know instantly if they are understanding the concepts.

3. Motivate students, allowing them to participate in class without the fear of making mistakes in public or making a fool of themselves.

4. Reward students who participate more in class or who best make progress.

5. Create balanced groups in class that bring together the most hardworking students and the least.

6. Improve the teacher-student relationship through a map of the class, which will allow the teacher to know the name of the students at all times and thus make a more personalized communication.

7. Register attendance to class automatically.

\subsection{Technologies}

Once the objectives are defined, the technologies, development environment and architecture of the system will be explained.

For the development of the application on the server, we have chosen the MEAN open-source environment, a full-stack JavaScript solution that helps building fast, robust, and maintainable web applications using MongoDB, Express, AngularJS, and Node.js. As shown in the Fig. 1, on the server side there is Node.js, an execution environment for event-driven server-side and networking applications, Express.js, a web application framework that runs on Node.js, and MongoDB, a NoSQL database. On the user side, we use Angular.js, a JavaScript MVC frameworks that run in browser JavaScript engines. The user application in the encapsulated in a mobile app (Android or iOS) using Cordova, a mobile application development framework that wraps HTML5 code into a multi-platform mobile app.

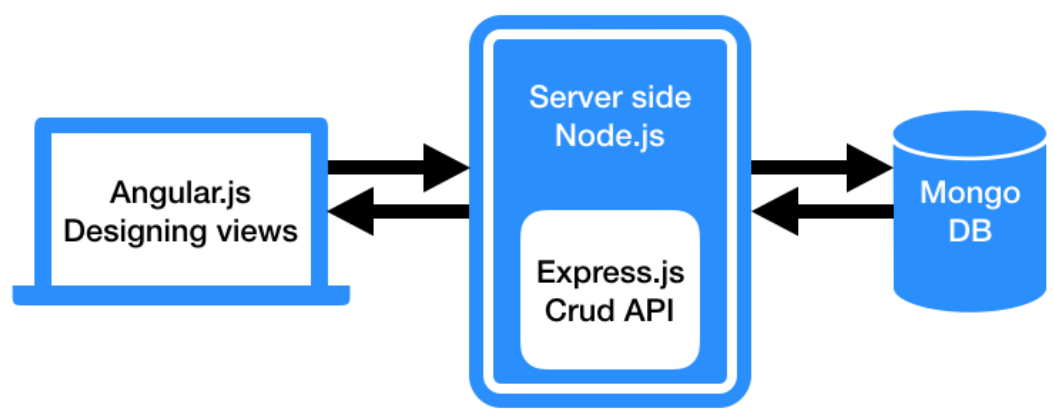

Figure 1. System overview

On the user side, there are two different profiles: lecturer and student. It is easy to select them by using user/password login screens.

Nowadays, there are many choices when it comes to coding and simplifying certain functions of the use of a mobile device. Currently existing technology allows us to insert information in certain labels or markers, in order to develop functions in the smartphone in a predefined way. 
Initially, two options were considered for this project: NFC and QR.

1) NFC (Near Field Communication) is a communication technology for proximity between devices without the need for cables or wires, conveniently and safely, just a few centimetres away. This technology offers a series of advantages: better visibility regardless of the light of the stay, longer life, gives the guarantee of the presence of the user and is easy to use.

2) A QR code is a square two-dimensional bar code that can store the encoded data and can include URL, vCard, text, email, SMS, social networks, PDF, MP3, APP stores, images, telephones, events, Wi-Fi and geolocation.

Since some smartphones don't have NFC at the moment, we have chosen QR codes.

\subsection{System Overview}

Fig. 2 shows the architecture of the system. On the one hand, there is a server with a database that is connected to the Local Area Network (LAN) of the University. On the other hand, there are the applications of the lecturer and students:

- The student has an app installed on his mobile phone, either Android or iOS. This application uses the camera to read the QR code that is installed in each of the tables in the classroom.

- The teacher accesses his application through a web browser, either from a mobile / tablet or from a computer.

The system forces both applications to connect through the university network, which avoids possible identity theft attempts.

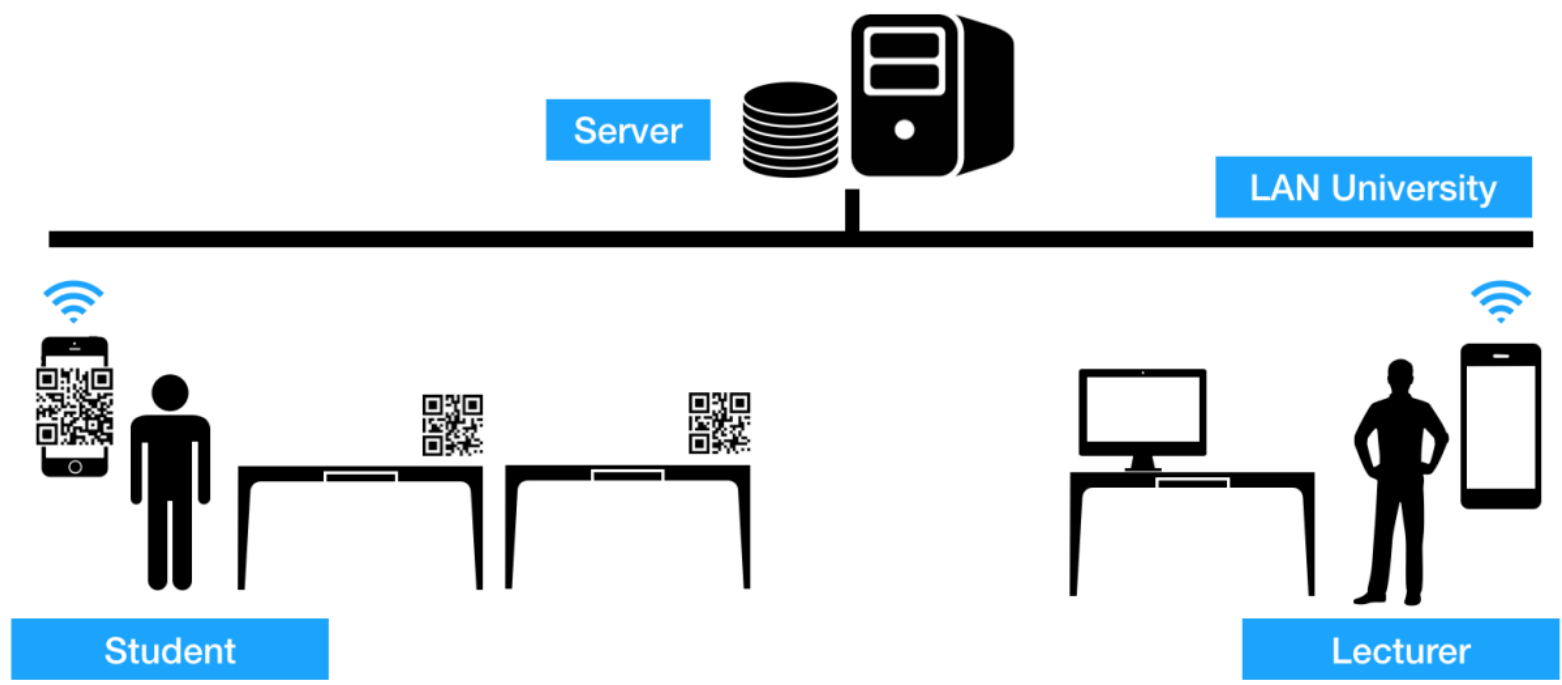

Figure 2. System overview

\subsection{User stories}

Two user stories have been defined to implement the application: one for the lecturer, and one for the student.

The student user story is as follows:

- As a student, I want to register ("check-in") in that class with my mobile device through the QR code located on my desk.

- I want the app to notify me when there is a test that the teacher formulates.

- I want to be able to answer the test from the app. 
- I want to be able to see the statistics of the questions-answers that I have done in each of the subjects.

The lecturer user story is as follows:

- As a lecturer, I want to visualize the map of the class, with names, photos and location of each student.

- I want to use the map through a tablet or PC to know the names of the students and to be able to interact on a personal level (this is especially relevant for classes of 100 students or more).

- I want to ask questions, tests, games, exercises, etc. that students answer individually and confidentially through their app.

- I want to visualize the statistics of each of the questions after having given a short time for the students to answer during the class.

- I want to see the rankings of the students, aggregated grades, and number of times they have not attended class.

Student responses can be used to make rankings or classifications of students, which can be used by the lecturers at their convenience, for example to award prizes according to the performance of each student (gamification techniques are used).

\section{RESULTS}

In this section an example of use is presented. The application has been designed to be used by one lecturer and several students at the same time. There is a different interfaces for the lecturer and the student, as it can be seen in the Fig. 3 .
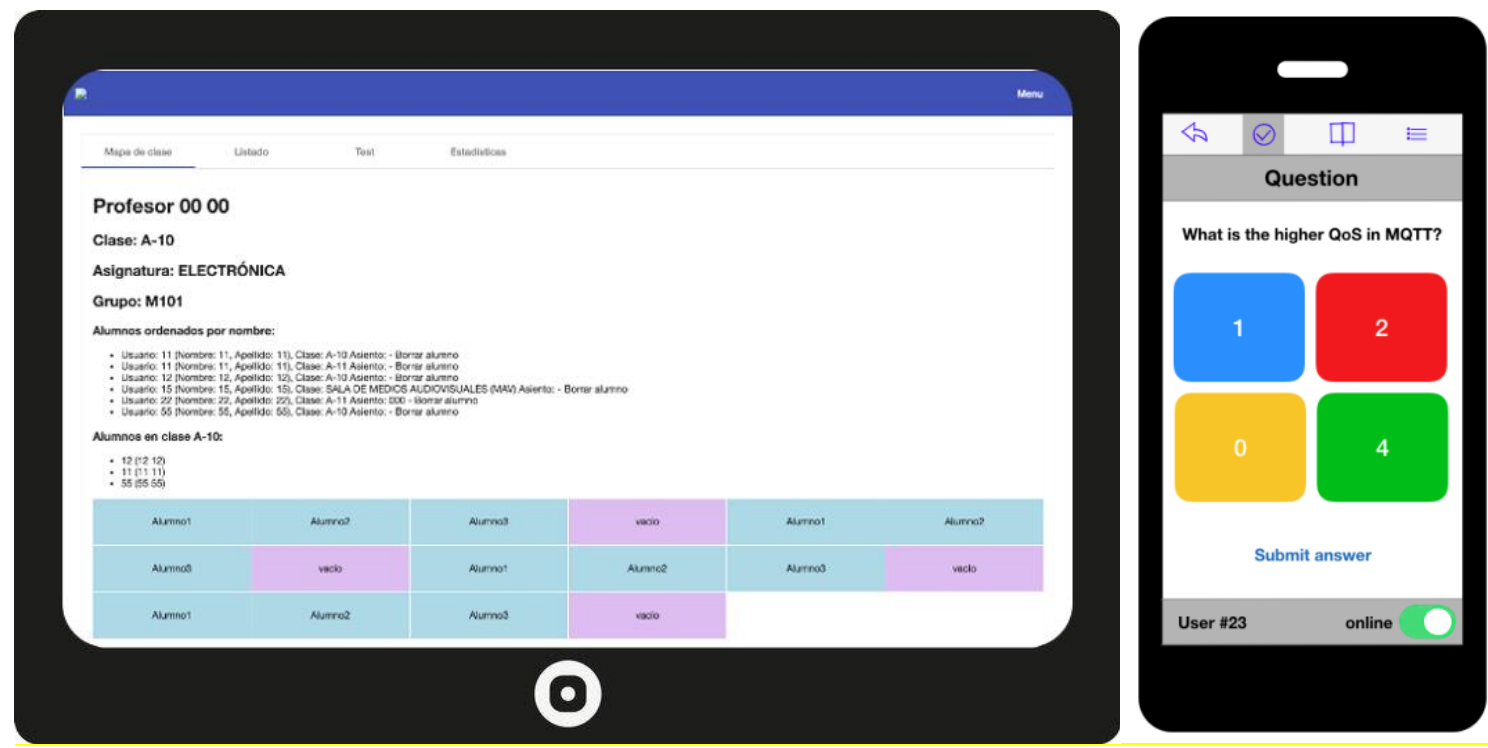

Figure 3. Lecturer's (left) and student's (right) interfaces

The lecturer's flowchart can be seen in Fig. 4 and the student flowchart in Fig. 5. The lecturer has to create a session for each lecture to allow students to log in. At any moment, the lecturer can create a new question (test, short question) by typing the question on the application. One the question has been submitted, student will receive an alert to answer the question.

Lecturers can see a map of the class in the app, so they can check how many correct answers there are. Both lecturers and students can check rankings and statistics to keep track of their progress.

Every time the student logs in the application by scanning the QR code, he/she attendance is registered for the record. 


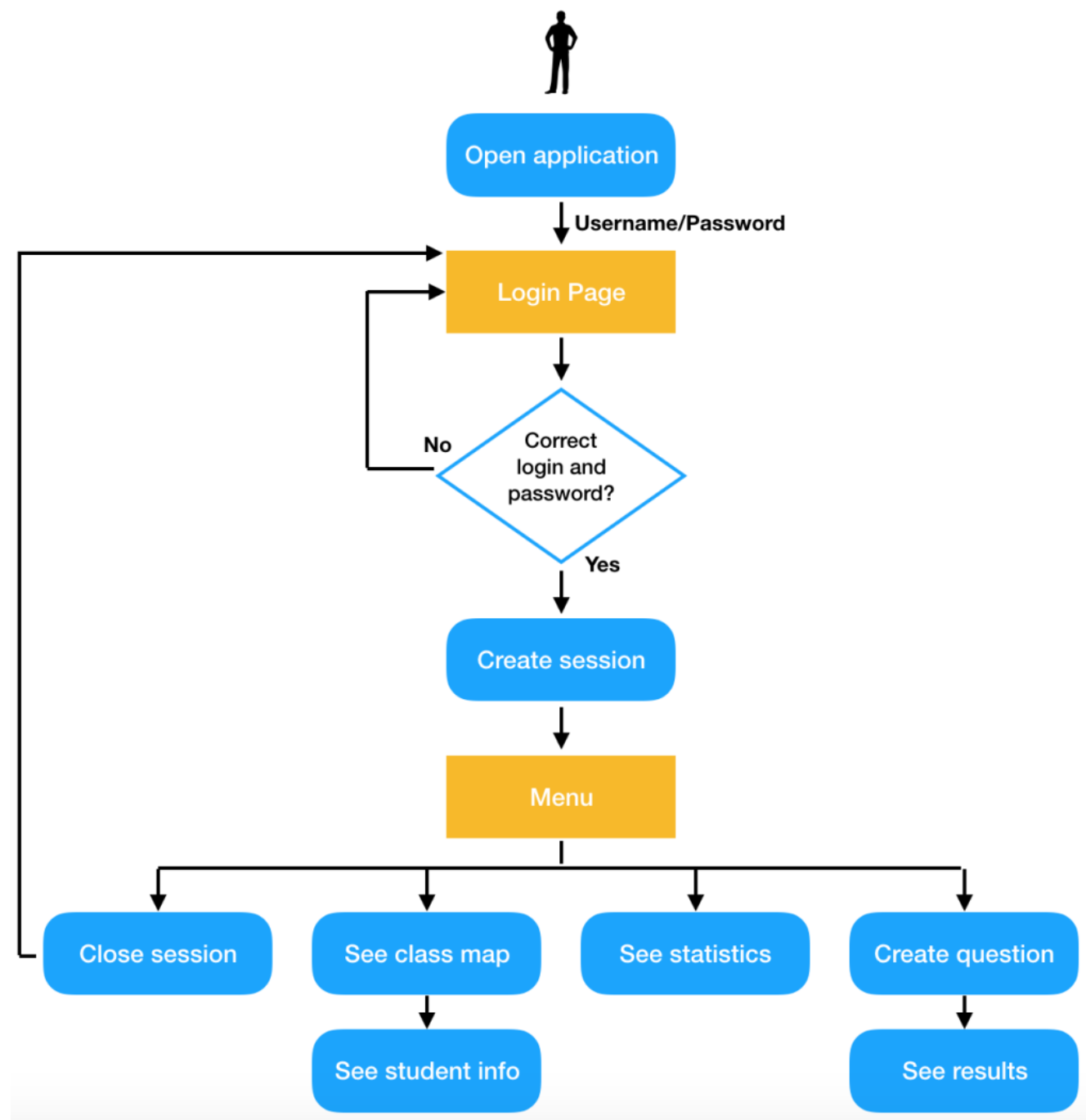

Figure 4. Lecturer's flowchart

\section{CONCLUSIONS}

In this paper a new idea to improve the user experience in face-to-face classes, both for lecturers and students has been presented. An app allowing the lecturer to have more control over the class, registering and visualizing the student attendance and performance, and allowing students to participate more actively during the sessions through live questions, has been designed.

This project contributes to improve both academic results and class activities. Regarding the improvement of academic results, the proposed system allows a constant and progressive evaluation of the students in each class. The lecturer can make questions directly to the students (without the need to prepare them in advance), and their answers will be recorded. This allows a progressive evaluation throughout the course, fairer in certain areas than the partial exams.

And regarding class activities, we believe that there is a clear problem of student participation in classes. Students are afraid to speak in class and to speak in public. Although public speaking is a competence that students must have, while they acquire it, this system allows them to participate in classes. This lack of participation means that lecturers do not get feedback from the students and do not know if what they are explaining is being understood or not. 


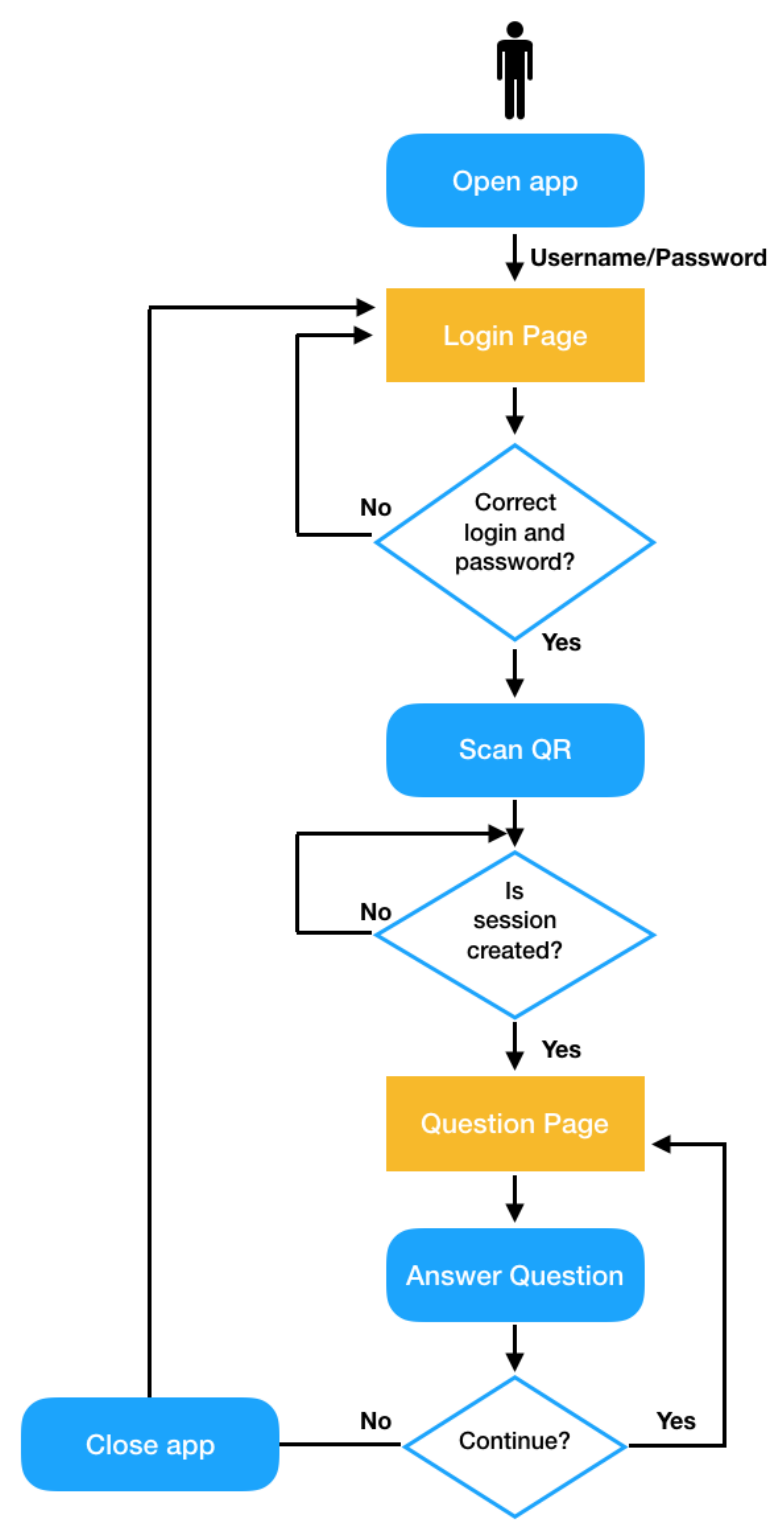

Figure 5. Student's flowchart

In some of the modules in which this app is to be implemented, there is a clear problem of absenteeism. We believe that this tool can be useful to motivate students by making classes more interactive.

The economic investment to implement this is minimal since QR codes can be printed and placed on tables for very little money and applications are installed on the devices of the students and lecturers themselves. Only one server PC is needed and the configuration of the system to the school or classroom. Therefore, it is believed that it will be a perfectly adaptable tool for any teaching classroom.

The application will be launched during the 2018-2019 academic year in the subjects taught by the authors' department. However, we still do not have performance results yet since we are at the beginning of the academic year and the last tests are being carried out.

\section{ACKNOWLEDGEMENTS}

This project has been funded by the Universidad Politecnica de Madrid within the "Aid for educational innovation and the improvement of the quality of teaching" during the academic year 2017-2018. 


\section{REFERENCES}

[1] Y. Chaiyo, R. Nokham, et. al, "The effect of Kahoot, Quizizz and Google Forms on the student's perception in the classrooms response system", International Conference on Digital Arts, Media and Technology (ICDAMT), 2017.

[2] C.M. Plump, J. LaRosa, "Using Kahoot! in the Classroom to Create Engagement and Active Learning: A Game-Based Technology Solution for eLearning Novices”, Management Teaching Review, vol. 2, no. 2, pp. 151-158, 2017.

[3] J. M. Krause, K. O'Neil \& B. Dauenhauer, "Plickers: A Formative Assessment Tool for K-12 and PETE Professionals", Strategies, 30:3, 30-36, 2017.

[4] Thomas, J., et al. "Participation and knowledge through Plickers in high school students and its relationship to creativity." UNESCO-UNIR ICT \& Education Latam Congress, 2016.

[5] MEI, Suo Yan; JU, Suo Yan; ADAM, Zalika. "Implementing Quizizz as Game Based Learning in the Arabic Classroom". European Journal of Social Science Education and Research, vol. 5, no. 1, pp. 208-212, 2018. 